\title{
Vérification systématique de la médication, test en situation
}

\author{
Liat Fishmana, Chantal Zimmermann ${ }^{\text {b }}$ \\ a Dr med., Responsable "progress! La sécurite de la médication aux interfaces», Sécurité des patients Suisse \\ ${ }^{b}$ M.A., Gestionnaire de projet, Sécurité des patients Suisse
}

Précisions sur le programme pilote national «progress!»: pour le projet d'approfondissement du programme pilote national "progress!» au début du programme, Sécurité des patients Suisse a fait confirmer par la Commission cantonale d'éthique de Zurich que la réalisation du projet était sans risque du point de vue éthique, même les relevés de données.

Pour faciliter la lecture du document, le masculin générique est utilisé pour désigner les deux sexes.
Les transferts de patients entre secteurs de soins entraînent des risques au niveau de la médication. A l'hôpital, l'admission et la sortie sont des moments particulièrement délicats à cet égard. De nombreuses études, réalisées dans différents pays dont la Suisse, montrent que l'anamnèse médicamenteuse est souvent incomplète à l'entrée [1]. De ce fait, sur la prescription hospitalière, certains médicaments disparaissent et d'autres sont ajoutés à tort, ou la posologie est mauvaise. Les traitements médicamenteux des patients étant souvent adaptés lors de leur séjour à l'hôpital, la sortie est aussi une interface critique pour la sécurité de la médication. Si les équivalences avec les anciens génériques ne sont pas rétablies, il y a risque de confusion pour les patients. Les pharmacies publiques et les médecins de famille reçoivent des prescriptions de sortie ou des listes des médicaments à prendre après la sortie, qui n'indiquent le plus souvent pas clairement les médicaments ajoutés, supprimés ou dont la posologie a été modifiée, ni les raisons de ces changements. Les doutes et les erreurs sont inévitables.

\section{Résumé}

Les transferts de patients entre secteurs de soins entraînent des risques au niveau de la médication. Une vérification systématique de la médication aux interfaces, lors de l'admission à l'hôpital, aux transferts internes et à la sortie de I'hôpital, réduit le pourcentage d'erreurs médicamenteuses et rend plus efficaces les processus de vérification. Le programme pilote national «progress! La sécurité de la médication aux interfaces», lancé dans ce but par l'Office fédéral de la santé publique, développé et mis en œuvre par la fondation Sécurité des patients Suisse, a débuté en mars 2015. Une campagne et un projet d'approfondissement, avec neuf hôpitaux pilotes dans toute la Suisse, en sont d'importants éléments. Sur la base de modèles de rôles variables, les hôpitaux pilotes testent la mise en œuvre de la meilleure anamnèse médicamenteuse possible dans chacun des services pilotes internes. Le présent article décrit le projet d'approfondissement et les premières expériences. Dans le cadre d'une problématique pratique du programme, Sécurité des patients Suisse a analysé, dans une étude séparée, le rôle des officines, qui ne sont quasiment pas utilisées comme source d'informations lors de l'admission des patients à l'hôpital pour connaître les médicaments qu'ils prennent. Pourtant, comme le montre l'étude explorative, elles pourraient fournir des informations importantes venant compléter l'anamnèse médicamenteuse, notamment pour leur clientèle d'habitués.

\section{Une vérification systématique de la médication réduit les erreurs médica- menteuses aux interfaces}

Dans les soins quotidiens, afin d'éviter ces erreurs médicamenteuses, dans de nombreux pays comme le Canada, les Etats-Unis, l'Angleterre et les Pays-Bas, il est procédé à une vérification systématique de la médication (en anglais: Medication Reconciliation, en français aussi: conciliation médicamenteuse) aux interfaces. Cela couvre l'établissement systématique d'une liste complète des médicaments et préparations qu'un patient utilise actuellement, ainsi qu'un recours automatique à cette liste dès que des médicaments sont prescrits. Cela permet de garantir que les indications relatives à la médication sont enregistrées et communiquées avec précision, exhaustivité et cohérence à toutes les interfaces de soins. Avec la mise en œuvre du programme pilote national «progress! La sécurité de la médication aux interfaces», la fondation Sécurité des patients Suisse souhaite favoriser l'application incontournable de la conciliation médicamenteuse dans les hôpitaux suisses (voir encadré à la page suivante).

Le programme pilote a été lancé en mars 2015, avec la publication des recommandations «Vérification systématique de la médication dans les hôpitaux de soins aigus», document à la disposition gratuite de toute personne intéressée. Le projet d'approfondissement a commencé en même temps dans les hôpitaux pilotes: il est axé sur la première étape de la vérification de la médication lors de l'admission, à savoir l'exécution de la meilleure anamnèse médicamenteuse possible (voir graphique). Car l'enregistrement complet et précis de la médication préhospitalière constitue la base d'une prescription d'entrée sûre à l'hôpital, et d'une vérification efficace de la médication à toutes les interfaces. $\mathrm{Au}$ moins deux sources d'informations sont mises à contribution pour recueillir le plus exhaustivement possible les renseignements sur la médication. L'une d'entre elle consiste en un entretien structuré des patients et/ou de leurs proches, quand c'est possible. Au cours de l'entretien, un guide doit être utilisé comme aide-mémoire, afin d'enregistrer le plus systématique- 


\section{Le programme pilote «progress! La sécurité de}

\section{la médication aux interfaces»}

Dans le cadre de la "Stratégie fédérale en matière de qualité dans le système de santé Suisse» entre 2014 et 2016 la fondation Sécurité des patients Suisse applique le programme pilote national "progress! La sécurité de la médication aux interfaces». II a été lancé et financé de façon déterminante par l'Office fédéral de la santé publique et développé par Sécurité des patients Suisse. Il est axé sur l'application de la vérification systématique de la médication dans les hôpitaux de soins aigus. Les contenus du programme sont inspirés de programmes réussis dans d'autres pays et sont adaptés au contexte suisse. Le programme est articulé autour de deux points forts:

1. Sensibiliser et informer: Le programme vise une sensibilisation globale à la problématique et à l'approche interventionniste de la vérification systématique de la médication, dans les hôpitaux, chez les autres prestataires et dans la population en général.

2. Projet d'approfondissement interprofessionnel pour les hôpitaux pilotes: Dans neuf hôpitaux pilotes, la vérification systématique de la médication lors de l'admission à l'hôpital est introduite et appliquée sur deux ans, dans des services médicaux sélectionnés.

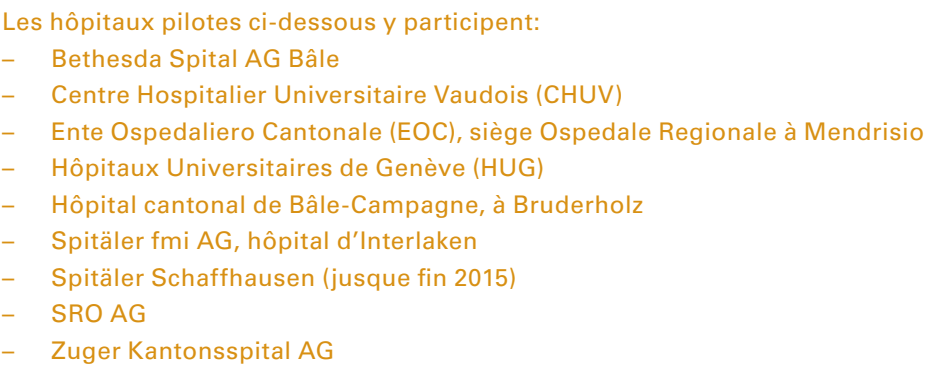

Des informations complémentaires sur le programme pilote sont consultables sur le site web de Sécurité des patients Suisse (www.securitedespatients.ch).

ment possible toutes les informations importantes. Ce guide contient par exemple des questions sur les médicaments et les préparations qui sont souvent oubliés, à savoir les médicaments sans ordonnance, les gouttes, les inhalateurs, les patchs,les pommades, les préparations végétales, les compléments alimentaires et les médicaments de réserve pour traiter la douleur, la constipation et les troubles du sommeil.

\section{Différents modèles de rôles}

Après une phase de préparation, les hôpitaux pilotes ont démarré la mise en œuvre des nouveaux processus fin 2015: chaque hôpital réalise, dans un service pilote avec des patients de médecine interne, 24 heures au plus tard après l'admission des patients, la meilleure anamnèse médicamenteuse possible, suivant les exigences de procédure définies dans le projet, et documente cette anamnèse avec un formulaire standard. Les hôpitaux ont défini les responsabilités du processus en fonction des réalités et ressources locales. Dans quatre hôpitaux, l'anamnèse médicamenteuse reste la tâche des médecins-assistants. Dans les autres hôpitaux, les assistants en pharmacie et les pharmaciens ou pharmaciens cliniciens dirigent cette consultation systématique des patients sur leur médication actuelle. Dans certains hôpitaux, les pharmaciens sont de plus sollicités pour comparer la liste des médicaments établie avec la prescription et pour procéder à d'autres contrôles, celui des interactions par exemple. Le recours aux pharmaciens et aux assistants en pharmacie pour établir l'anamnèse médicamenteuse et valider les prescriptions est déjà une réalité partielle depuis des décennies dans d'autres pays, dont ceux mentionnés auparavant. Selon la littérature actuelle, la vérification systématique de la médication s'avère plus efficace lorsqu'elle bénéficie d'une participation active du personnel de la branche pharmaceutique. Le projet «progress!» permet désormais de tester ces modèles de rôles alternatifs également dans le contexte suisse. Quelle que soit l'attribution des tâches, il est essentiel que le personnel spécialisé dispose d'assez de temps. En effet, l'anamnèse systématique de la médication, sur la base d'un entretien structuré avec le patient, et d'une comparaison précise de plusieurs sources, demande plus de temps que l'anamnèse traditionnelle. Tous les participants doivent en être préalablement conscients. Mais cet investissement temps vaut la peine, car il raccourcit et facilite les processus suivants, ce qui permet d'augmenter globalement l'efficacité et la sécurité de la médication.

\section{Les officines comme source d'information}

D'après le Moniteur des pharmacies 2014, 80\% de la population suisse garde la plupart du temps la même pharmacie, qui est en quelque sorte leur pharmacie habituelle [2]. On peut donc supposer que les officines disposent d'informations utiles pour une anamnèse médicamenteuse lors de l'admission à l'hôpital. Au Canada, en France et aux Pays-Bas, elles jouent déjà un rôle central dans le relevé de la médication préhospitalière. Afin d'analyser dans quelle mesure les officines de notre pays peuvent être utilisées comme source d'information par les hôpitaux, et quelles informations elles peuvent contribuer à un pointage exhaustif des médicaments, Sécurité des patients Suisse a mené en 2015 une enquête qualitative financée par pharmaSuisse. Onze pharmaciens de six cantons différents ont été interviewés. En outre, dans le cadre d'une thèse à l'université de Bâle, deux questions ont pu être posées dans un questionnaire en ligne, et envoyées à toutes les administrations des pharmacies enregistrées auprès de la Société Suisse des Pharmaciens. Etant donné le faible taux de retour (inférieur à 15\%), les résultats doivent cependant être interprétés avec prudence. 
Vérification systématique de la médication dans les hôpitaux de soins aigus



La vérification systématique de la médication se fait en plusieurs étapes, à chaque interface dans I'hôpital.

\section{Fondation Sécurité des patients Suisse La véri- fication systématique de la médication dans les hôpitaux de soins aigus. Recommandations dans le cadre du programme pilote national progress! La sécurité de la médication aux inter- faces 2015. Ce document peut être téléchargé à l'adresse: http://www. patientensicherheit.ch/ fr/publications Mat-riel-d-information- Publications.html Le document cité ici indique les études sur lesquelles s'appuie cet article. D'autres sources y sont aussi mention- nées. \\ gfs.bern. Moniteur des pharmacies 2014. Pres- tations de services oui, mais pas à n'importe quel prix. 2014 [consulté le 24 sept. 2014 ]. Ce do- cument peut être télé- chargé à l'adresse: http://www.gfsbern.ch/ de-ch/Detail/dienstlei tungen-ja-aber-nicht- um-jeden-preis}

Correspondance: Fondation Sécurité des patients Suisse Dr med. Liat Fishman Asylstrasse 77 CH-8032 Zurich fishman[at] patientensicherheit.ch
Les résultats de l'étude montrent que les hôpitaux n'utilisent que rarement, voire jamais, les officines comme source d'information, qu'elles se trouvent dans un secteur avec propharmacie ou non. Pourtant, les officines peuvent tout à fait disposer de renseignements importants sur la médication de leurs clients habitués, à savoir les prescriptions d'autres médecins traitants, des ordonnances de médication de sortie d'hôpital ou des produits complémentaires achetés à la pharmacie, des médicaments sans ordonnance par exemple. Les pharmacies peuvent aussi avoir des indications sur l'adhésion au traitement, comme des difficultés avec certains médicaments, des risques de mauvais comportements ou des achats abusifs. Les hôpitaux pourraient donc avoir intérêt à contacter la pharmacie habituelle de leurs patients à l'entrée de ces derniers, afin de connaître les médicaments pris avant l'admission. Cependant, les pharmaciens consultés ont bien précisé que leurs informations pourraient être lacunaires. Par exemple, ils ne savent pas avec certitude si les indications fournies sur la médication, que ce soit par le médecin ou par le client, sont exhaustives, et si leurs clients n'ont pas complété la quantité de médicaments ou n'en ont pas acheté d'autres dans d'autres points de vente. Mais les interviews individuelles et le sondage en ligne montrent que, notamment dans les secteurs sans délivrance de médicaments par les médecins, les pharmacies estiment que le niveau d'exhaustivité des informations fournies par leur clientèle habituelle est élevé.

Si les officines étaient plus souvent utilisées lors de l'admission à l'hôpital comme source d'information sur leur clientèle habituelle, il serait nécessaire de clarifier la transmission d'informations. Les estimations divergent notamment dans le domaine de la protection des données. Il faudrait définir quelles informations les officines peuvent transmettre, à qui, et comment. En outre, il résulte de l'étude que le flux d'informations doit avoir lieu dans les deux sens. Les pharmaciens interrogés ont notamment rencontré des problèmes avec les ordonnances de sortie d'hôpital. Elles mentionnent des ordonnances de sortie d'hôpital non claires, illisibles et incomplètes, ne tenant souvent pas compte de la médication préhospitalière. «Le cas classique est celui des génériques. A l'hôpital, ils changent tout, et au final le client avale plusieurs fois la même chose, car il ne sait pas que c'est pareil», raconte une pharmacienne. Pour garantir la sécurité de la médication, il serait judicieux d'améliorer la communication aux interfaces entre hôpital et pharmacie habituelle, dans les deux sens.

\section{Perspectives}

Le programme "progress! La sécurité de la médication aux interfaces» se poursuit jusqu'à la fin de l'année. D'ici là, la fondation Sécurité des patients Suisse accompagne les hôpitaux pilotes avec des relevés de données et des visites sur place, afin d'analyser de plus près les processus, avec des groupes de projet interprofessionnels. Le quatrième et dernier atelier du projet d'approfondissement aura lieu en décembre 2016. C'est une nouvelle occasion d'échanges d'expériences pour les groupes de projet. En outre, les résultats du projet seront présentés et débattus. L'objectif est qu'une fois le projet terminé les hôpitaux puissent intégrer aux soins quotidiens la vérification systématique de la médication et appliquer le processus jusqu'à la sortie du patient. Ce n'est pas une mince affaire, car des processus complexes, impliquant de nombreux participants, doivent être adaptés, ce qui suppose des ressources en personnel et en temps. Enfin, les expériences du projet d'approfondissement doivent profiter également aux autres hôpitaux suisses, lors de l'introduction et de la mise en œuvre de la conciliation médicamenteuse. Les résultats seront aussi présentés dans cette revue en temps et en heure.

\section{Crédit photo}

(c) Sécurité des patients Suisse

\section{Disclosure statement}

Aucun conflit d'intérêt.

\section{Précision}

Le rapport des résultats de l'étude sur les officines (en allemand) peut être consulté en ligne: http://www.patientensicherheit.ch/fr/th-mes/ Autres-th-mes-relatifs---la-s-curit--de-la-m-dication/Les-pharmacies-d-officine--source-d-information.html 